\title{
An update of molecular basis of telomere biology diseases
}

\author{
Eleonora Vertecchi, Angela Iuzzolino and Erica Salvati *
}

${ }^{1}$ Institute of Molecular Biology and Pathology, National Research Council, Rome, Italy, c/o Department of Biology and Biotechnology, Sapienza University of Rome, Via degli Apuli 4, 00185 Rome, Italy.

* Correspondence: erica.salvati@unitoma1.it

\begin{abstract}
Telomeres are crucial structures that preserve genome stability. Their progressive erosion over rounds of DNA duplication determines senescence of cells and organisms. In a classic view, telomere biology impinges on intracellular signaling pathways regulating DNA damage repair and cell cycle arrest, but new roles of telomeric proteins and transcripts emerge from recent literature. Telomere biology diseases are human disorders associated to telomere attrition. This review wants to overview the recent findings in the field of telomere's metabolism and to deepen molecular mechanisms of inherited and acquired telomeropathies, explaining new critical connections between telomeric factors and disease pathogenesis.
\end{abstract}

Keywords: telomeres, shelterins, telomere biology diseases, cancer, dyskeratosis

\section{Telomeres structure and function}

Telomeres are specialized structures at chromosome ends deputed to protection and preservation of genetic information. Evolutionarily, telomeres are deemed to originate from introns recombination in circular DNA molecules, generating non-coding extremities[1].The presence of terminal non-coding DNA overcame the progressive loss of genetic material at each cell division round, due to the incomplete replication of linear DNA molecules[2]. Telomeres are in fact constituted by non-coding repeated DNA. Telomere repeats are species-specific G-C rich conserved sequences, (in human 5'-TTAGGG- 3') that terminate with a G-rich (or in some species both $\mathrm{G}$ and C-rich) overhang[3]. The overhang is fundamental for telomere stability since its capacity to invade the duplex and to form a cap-like structure (the T-loop) is at the basis of telomere protection[4][5]. In fact, the loss of G-overhang was shown to cause a rapid induction of DNA damage response (DDR) in cells. To allow the cell to discriminate between induced DNA lesions and natural 
extremities, telomeres are covered by the shelterin complex. The complex is composed by six proteins: TRF1, TRF2 and POT1 bind telomeric DNA directly, while TPP1, TIN2 and Rap1 act as a bridge among the shelterin factors, holding fast the structure of the complex itself [6]. The member of the shelterin complex have distinct function directed to different DDR signaling and repair pathways [7] and are involved in regulation of telomere elongation [8].

When telomeres undergo massive erosion or genotoxic stresses causing loss of telomeric repeats, the protection complex is insufficiently bound, and DDR is activated leading to cell arrest and senescence[9]. Dysfunctional telomeres are recognized as DNA damage and repaired unproperly by Homologous Recombination and canonical or non-canonical NonHomologous End Joining, generating telomere aberration such as fusions or loss of telomeric repeats. In precancerous lesions, where cell cycle checkpoints that arrest cells upon DNA damage are altered, telomere instability generates mis-segregation and chromosome breakage during mitosis, giving rise to secondary rearrangements that fuel genetic instability[10]. Thus, telomere protection is considered a tumor suppressive element. Otherwise, telomere length maintenance is a pre-requisite for cancer development since telomere attrition during cell divisions must be buffered in actively replicating cancer cells, in order to maintain an unlimited proliferative potential. Cancer cells in fact re-activate telomerase enzyme or alternative mechanisms to elongate telomeres during cancer progression.[11]

It has been known from years that inherited telomere length is correlated with lifespan of cells and organisms, and that telomere length is reduced over age [12]. Telomere shortening and dysfunction is a hallmark of ageing, since telomere attrition causes stem cell exhaustion impairing tissue renewal. In addition, mounting evidence support a role for telomere's dysfunction in ageing pathologies where a critical role is played by inflammation [13]. Recently, an extensive analysis of telomere length (TL) in different human tissues types and individuals clearly showed a significant correlation of TL with genetic background, gene expression and ageing. Furthermore, this analysis clearly showed that telomere shortening mediates aging related gene expression [14]. For longtime telomeres have been considered as silent chromatin territories. Recently, telomeres were discovered to be transcribed into long non-coding RNAs (lncRNAs). Telomere transcription is associated with telomere stability, DNA damage response and aging. TERRA are lncRNAs transcribed from subtelomeric promoters which are involved in telomere stability. They are regulated by the methylated state of subtelomeres and are strongly upregulated in ALT cells. They remain associated to telomeric chromatin forming R-loops which increases the predisposition to hyper-recombination of ALT telomeres[15]. Upon DNA damage at telomeres, damage induced long non coding RNAs (dilncRNAs) are transcribed bidirectionally starting from the DNA lesions, they are precursor of small non coding RNAs (named DDRNAs) and mediate the efficient transduction of signalling cascade driving cell arrest and repair[16][17]. Transcription mediated telomere damage is also responsible for the expression of senescence associated cell phenotypes[18]. 
Telomeres are difficult to replicate regions, being constituted by heterochromatin and prone to fold into secondary structures like G-quadruplexes, t-loop, I-motifs[19]. In addition, the presence of long non-coding RNA transcribed from subtelomeric promoters, that stably interact with DNA duplex forming R-loops, [20] make this chromosome fields enriched of topological enzymes necessary to assist replication, transcription, histone modification. Telomeres are in fact considered as "difficult to repair" chromatin, that consequently accumulate irreparable DNA damage causing senescence and aging.[21] In this regard, mutations affecting helicase, topoisomerase, histone acetylation and methylation cause telomere's dysfunction and consequently aging associated phenotypes.

This review wants to overview and deepen molecular mechanisms of inherited and acquired telomeropathies, explaining new critical connections between telomeric proteins and disease pathogenesis.

\subsection{Telomere length homeostasis.}

Some cells, like gametes, cancer and stem cells, have developed a successful strategy to overcome the replication end problem through the expression of telomerase, a ribonucleoprotein involved in counteracting the shortening of telomeric ends. Telomerase expression is strictly controlled throughout human development: if embryo stem cells have a high activity of telomerase, in the most adult somatic cells telomerase is not detectable, with the exception of lymphocytes in bone marrow and peripheral blood and a cluster of epithelial cells in the skin, hair follicle, endometrium and gastrointestinal tract [8] [22] [23]. If mutations that make telomerase inefficient happen during the embryogenesis process, cells will be generated with short telomeres right from the beginning, making highly probable the occurrence of telomeropathy [24] [25].

Telomerase is a holoenzyme able to maintain telomeres length, re-synthesizing telomeric repetitions that are lost at each replication cycle [26]. It was discovered in 1985 in the ciliate Tetrahymena thermophila and it was called "telomere terminal transferase" to highlight its capacity to add telomeric sequence repeats [27]. Nowadays, human telomerase structure has been defined; it is a ribonucleoenzyme formed by hTERT, the reverse transcriptase that represents catalytic enzyme core, hTERC, the IncRNA used as a template for telomere elongation, and a series of specie-specific accessory proteins: dyskerin, NHP2, NOP10, reptin/pontin, Gar1 and TCAB1 (Fig.2) [28][29][30][31]. Accessory telomerase proteins regulate telomerase activity, biogenesis and localization and are involved in many biological processes [32]; dyskerin, for example, is a pseudouridine synthase localized mainly in the nucleus, where it can participate in the formation of telomerase, Cajal body ribonucleoparticles (scaRNPs) and H/ACA small nucleolar ribonucleoparticles (snoRNPs), playing an important role not only for telomeres, but also for rRNA processing [33]. 


\section{Telomeres and cancer}

Telomeres length maintenance is a pre-requisite for cancer development,[34] recently, a pan-cancer genomics study detected TERT expression in $\sim 75 \%$ of tumor samples. In these samples, telomerase reactivation occurred by point mutation (31\%) or methylation $(53 \%)$ in the hTERT promoter.[35] Activation of telomerase coincide with other pro-oncogenes changes in adult somatic cells in the early steps of cancer development [11]. The pro-oncogenic activity of telomerase is not restricted to telomere elongation but involves interactions between hTERT subunit and signaling pathways controlling cell survival and transformation like c-myc, WNT/ßcatenin, NF-kB, but the number of identified cross-talks between hTERT and intracellular signaling is constantly growing. [36] Telomerase reactivation mechanisms include promoter mutation and epigenetic modification, gene amplification, rearrangements of gene locus, [37] and enzymatic activity is directly correlated with cancer cell proliferation and stemness. [38] Other telomeric proteins are found mutated or deregulated in cancer. POT1(Protection of Telomeres 1) is an essential component for telomere stability, [39] it binds both the ds and the ssDNA at telomeres interacting with other shelterins (namely TPP1 and TRF1), counteracts G-quadruplex formation [40] and attenuates ATR driven DDR. Germline and sporadic mutation of POT1 are associated with different human cancers. POT1 is frequently mutated in aggressive forms of chronic lymphocytic leukemia. Furthermore, germline POT1 mutations have been shown to underlie a number of hereditary familial cancer syndromes involving CLL, glioma, melanoma and colorectal cancer and angiosarcoma. [41]. Telomere binding proteins are overexpressed in cancer, this is not simply explained by telomere re-elongation, in fact, some aggressive cancers present an unbalance between telomere length and telomere binding protein expression, which can be at the basis of the presence of dysfunctional telomeres generating genome instability [42] [43]. TRF1 is over-expressed in early stages of pancreas tumorigenesis and glioblastoma progression in mouse models [44] and TRF1 SNPs were found associated with increased risk of skin cancer in human[45]. TRF1 is considered a validated target in gliobastoma treatment and TRF1 inhibitors are currently under pre-clinical evaluation [46]. TRF2 is upregulated in several human cancers, it is involved in angiogenesis through different pathways and immune escape[47][48][49][50]. Recently, alterations of the shelterin complex were recently assessed in 9125 tumor samples in 33 different human cancers. TRF1 and POT1 amplification and TRF2-RAP1-TPP1 co-amplification/deletion were found associated with cancer progression defining broad molecular signatures linked to several intracellular pathways involved in oncogenesis[51]. Data collected in endometrial cancer patients suggest instead an inverse correlation between TERRA expression and cancer progression[52]. 


\section{Telomeropathies}

Telomeropathies are divided into two groups:

- Primary telomeropathies are a spectrum of telomere biology disorders occurring when genes that preserve telomeres are mutated. This category is represented by three different forms of DC: Hoyeraal-Hreidersson syndrome, Revesz syndrome and Coats Plus syndrome;

- Secondary telomeropathies are characterised by mutations on proteins involved in DNA damage response (DDR), such as Fanconi anemia, Werner syndrome, Rothmund-Thomson syndrome and Bloom syndrome [53][54].

\subsection{Dyskeratosis congenita}

Dyskeratosis congenita (DC, also referred to as Zinsser-Engman-Cole syndrome) is a rare disorder (prevalence: 1 case in 1 million people) characterized by a spectrum of symptoms like reticular hyperpigmentation, nail dystrophy, unusual skin pigmentation, eye and dental anomalies, oral leukoplakia [55] and cancer predisposition that affect cells with highturnover progenitor [56] [57] [58]. DC's patients have in fact a high probability to develop solid tumours and leukaemia [59] and usually death occurs for bone marrow failure (BMF) or pulmonary disorders [60].

The genetic basis of DC are to be found in mutations on key genes for telomere maintenance and for telomerase synthesis and activity [61] that cause in DC's patients a shorter telomere length than healthy individuals [62]. It presents three possible patterns of inheritance, according to which gene is mutated: X-linked, autosomal dominant or autosomal recessive [63] [64]. DC is also classified as ribosomopathy, because DC's patients present dysfunctions in ribosomes' synthesis and activity [61].

Generally, DC affects males three times more commonly than females because the more frequent mutation occurs on DKC1 gene on X-chromosome, linked to X-recessive inheritance [58] [65]. DKC1 encodes for dyskerin, a pseudouridine synthase involved in RNA modification [66]. Dyskerin is a member of H/ACA box snoRNPs family that includes ribonucleoprotein complexes formed by one H/ACA snoRNA and four proteins: dyskerin, GAR1, NHP2 and NOP10 [67]. When rRNA target must be modified, H/ACA snoRNA binds target throughout sequence-specific base pairing and dyskerin enzyme is activated, allowing rRNA maturation and functioning [67].The protein components of H/ACA snoRNPs complexes are also important because they constitute together with hTERT and hTERC another H/ACA RNP complex called telomerase (Fig.2) [66].

DKC1 was the first discovered gene involved in dyskeratosis congenita [68], but nowadays we know 10 other genes, whose mutations have a direct or indirect impact on telomeres length: TERC, TERT, NPM1, TRF1-interacting nuclear factor 2 (TINF2), adrenocortical 
dysplasia (ACD), conserved telomere maintenance component 1 (CTC1), NHP2, NOP10, poly(A)-specific ribonuclease (PARN), regulator of telomere length 1 (RTEL1) and WRAP53 [69] [64]. We can divide these genes into five groups (Table 1):

- Telomerase genes. TERC and TERT are two genes that encode, respectively, for telomerase RNA hTERC and for telomerase reverse transcriptase. NHP2 and NOP10, instead, are two accessory proteins of telomerase RNP complex involved also in biogenesis and functioning of rRNA [70] [71]. Finally, WRAP53 codes for the accessory protein TCAB1, that regulates telomerase trafficking and DKC1, whose function has been explained above [72];

- Telomerase related genes. CTC1 is a protein of CST complex, involved in telomerase activity, acting as a terminator of the reverse transcription process [73]. PARN is a specific exonuclease for poly-A RNA tail involved in hTERC processing, deadenylating hTERC to increase its stability [74] [75];

- Shelterin complex genes. ACD is a gene on chromosome 16 that encodes for TIN2interacting protein 1 (TPP1). TPP1 is a member of the shelterin complex that forms a bridge between POT1 and TIN2 [76]. Its main activity is telomeres maintenance [77], making possible shelterin complex assembling [78]. TINF2, instead, encodes for TIN2, the central component of shelterin complex that connects TRF1, TRF2, and POT1, regulating telomere structure maintenance and signalling [79];

- Shelterin related genes. RTEL1 encodes for a DNA helicase that interacts with TRF2 by facilitating telomeres replication [4].

- Ribosome related genes. NPM1 is a phosphoprotein involved in regulation of rRNA synthesis and maturation. Recently, it was demonstrated it is also implicated in maintenance and organization of nucleoli [80].

In a non-pathological situation, telomerase is activated right after fecundation, at blastocyst stadium [81] and it remains functional during embryogenesis until birth [81] [82]. Further, embryo expresses shelterin complex to protect its telomeres [83]. This creates pools of adult cells with a correct telomere's length, ready to shorten at every replicative cycle up to replicative senescence [84].

Table 1. Dyskeratosis congenita mutations summary

\begin{tabular}{ccc}
\hline Group number & Group name & Genes involved \\
\hline 1 & Telomerase genes & TERC, TERT, NHP2, NOP10, WRAP53, DKC1 \\
\hline 2 & Telomerase related genes & CTC1, PARN \\
\hline 3 & Shelterin complex genes & ACD, TINF2 \\
\hline 4 & Shelterin related genes & RTEL1 \\
\hline
\end{tabular}


In DC's patients, mutations on the above-mentioned key genes for telomeres maintenance generate individuals with already genetic instability and short telomeres at birth, causing serious problems that manifest as dyskeratosis congenita symptoms and cancer predisposition [85].

\subsection{Dyskeratosis and ribosome biogenesis}

\subsubsection{Ribosome biogenesis}

Human ribosomes (90S) are riboprotein structures composed by two subunits, small subunit (40S) and large subunit (60S), which play the role to scan and translate mRNA into proteins[86]. At molecular level, ribosomes consist of 80 ribosomal proteins (RPs) and 4 ribosomal RNA (5S, 5.8S, 18S, and 28S rRNA), transcribed into a nuclear specialized zone called nucleolus. Nucleoli are assembled around rRNA genes (rDNA), that are organised as head-to-tail tandem repeats set on chromosomes 1, 13, 14, 15, 21 and 22 [80] [86] [87]. rRNA transcription is regulated by many factors and one of the most important is nucleophosmin 1 (NPM1), which is responsible for histone distribution on rDNA [80] but it also has got a role in post-transcriptional modifications of rRNA [69]. During transcription rRNAs start to be modified by indispensable processes that carry out rRNA maturation. These processes are extremely regulated by several catalytic complexes:

- pre-5S is transcribed by tandem repetitions on chromosome 1 and needs $5^{\prime}$ and $3^{\prime}$ exonuclease trimming to generate mature $5 \mathrm{~S}$ rRNA, which will become part of $60 \mathrm{~S}$ ribosome subunit [88]. It is important to note that pre-5S is the only rRNA transcript synthetized out of nucleolus [89];

- $\quad$ pre-47S is processed by several nucleases to form 5.8S, $28 \mathrm{~S}$ and $18 \mathrm{~S}$ rRNAs. Furthermore, two snoRNPs, C/D and H/ACA, insert 2'-O-methylation and pseudouridylation to permit final rRNA maturation [61][88] .

\subsubsection{Dyskeratosis as a ribosomopathy: which is the link?}

For a long time it was thought that dyskeratosis congenita was provoked exclusively by mutations on dyskerin, which, being no longer functional, did not allow the correct assembly of one of the most important complex that contains it: the telomerase [68] [90].Today it is known that there are many mutations that result in DC and they can be related to telomerase complex, shelterin complex or ribosome biogenesis malfunction [43].

At this time, we might wonder about what type of molecular mechanism lies behind these mutations: can we report in all a reduction of telomerase activity? Or come into play 
another mechanism that can help us to understand the molecular pathways of dyskeratosis congenita? Especially, why is dyskeratosis congenita considered a ribosomopathy? What sort of role ribosomes have in this disease?

To answer to these questions we started from a piece of data of which we have a match in all DC's forms: patients 'cells, especially those with a high turn-over, present a telomere shortening and a ribosomes 'synthesis dysfunctions [61] [91]. These two structures, telomeres and ribosomes, that seem to be very far from a localization point of view, are related from a biogenesis point of view. Telomeres, in fact, are lengthened by telomerase enzyme which, as ribosomes, is a riboproteic structure formed by proteins and RNA, hTERC, processed by H/ACA box snoRNP [31]. Further H/ACA snoRNP and telomerase are linked by the presence of proteins dyskerin, GAR1, NOP10 and NHP2 [67] [56].

The conclusion to which we might arrive is that mutations in genes encode for either telomerase complex or H/ACA box bring to a malfunctioning of these complexes, with consequent failure of telomeric elongation and maturation of key rRNA. This may be true for all those dyskeratosis congenita forms generated by mutations on first, second and fifth classes of genes listed above, however it cannot explain telomerase or ribosomes dysfunction in patients with mutations on genes encode for shelterin protein or shelterin-associated proteins.

So, we have started to analyze the telomeres shortening in DC 'cells as starting point that unites all forms of dyskeratosis congenita:

- cells with first or second group mutations have a faulty telomerase, that cannot elongate telomeres as wild type one;

- cells with third or fourth group mutations have a defect on one of the components of shelterin complex and this leads to a failed recruitment of telomerase, that, as was seen in some papers, is called by dyskeratosis congenita shelterin mutated components [3] [92] [93] [94];

- cells with fifth group mutations have defects on NPM1. It was recently discovered that wt-NPM1 can associate with AP-endonuclease 1 (Ape1), regulating nuclease activity on telomeres. Because mutations on NPM1 cause a decrease of working NPM1, the presence of NPM1 in a sub-stoichiometric ratio respect to Ape1 carries out to the endonuclease activation and telomeric shortening [95].

At this point, we hypothesized that in all cases of dyskeratosis congenita it could play a role a molecule directly or indirectly involved in shortening of telomeres and in deregulation of protein synthesis. Accumulating evidence shows that telomere binding proteins such as TRF1, TRF2 and RAP1, bind extra-telomeric DNA regions, implying they have a function apart from the classic one [96]. 
By analyzing these proteins localization, our attention was focused on TRF2 because it is abundant into nucleolus, site of rRNA synthesis [97] [88] [98]. Moreover, it binds to nucleolar proteins and is involved in rRNA transcription. In dyskeratosis patients, unbalance between TRF2 protein levels and its preferential target (telomeric repeats) could increase TRF2 extra-telomeric localization affecting nucleolar structure and function and influence ribosome biogenesis.

\subsection{Dyskeratosis congenita: towards new therapeutic horizons}

Dyskeratosis congenita is an extremely heterogeneous pathology not only because, as it was seen, it is provoked by mutations on different genes, but also because, by a clinical point of view, it presents with very different symptoms from one patient to another and it strikes several tissues and organs [59]. Among the most common symptoms are: reticular skin pigmentation, nails dystrophy, aplastic anemia, bone marrow failure, leucoplakia, pulmonary diseases, learning disorders, mental retardation, extensive dental problems and tumors predisposition [99] [100] [60].

Several clinical tests exist to evaluate if a patient is affected by dyskeratosis congenita including the measurement of telomeres length by flow cytometry and fluorescence in situ hybridization as well as genetic tests for known mutations [101].

Today dyskeratosis congenita is not curable, but it can be treated acting on the many symptoms it produces [102].

Hematopoietic staminal cells (HSC), fundamental for daily production of blood cells, express telomerase so problems on functioning of this enzyme generate several symptoms as aplastic anemia and successively bone marrow failure [103]. Generally aplastic anemia is treated with frequent blood transfusions and, waiting for bone marrow transplant, patients undergo treatment with granulocyte macrophage colony-stimulating factor [104]. $\mathrm{BMF}$, instead, does not respond to immunosuppressive therapy, so the only possible treatment is hematopoietic stem-cell transplantation (HSCT), which, nevertheless, often results in pulmonary fibrosis that leads to death. For this reason, HSCT is often accompanied by lung transplant [105].

A therapy used to avoid bone marrow failure involves the use of drugs that activate TERC locus in HSC, but they can also be exploited by tumoral cells, often present in DC's patients, promoting progression of tumor itself [106].

Another therapy is based on androgens because telomerase can be modulated by sex hormones. Androgenic therapy seems to have a good response in hematologic recovery of red blood cells and platelets, even if it leads to a strong lipidic anomaly[102][107].

In the last years it has been studied a therapy that involves the use of sirtuins, a class of proteins involved in cell cycle regulation, DNA reparation, apoptosis and cellular survival 
[108]. SIRT6 is a possible DC target, preserving telomeres by senescence and senescent chromosomal aberrations [109]. If this therapy based on SIRT6 is truly effective, it would be in support of model proposed above: it is known, in fact, that SIRT6 plays an important role on TRF2 regulation and SIRT6 increasing generates an increasing of SIRT6-TRF2 interaction, that provokes degradation of this latter [110].

\section{Secondary telomeropathies, what is new?}

Secondary telomeropathies originate from inherited or acquired mutation of genes, which in turn affect telomere length and function. All these pathologies are characterized by aging marks and cancer predisposition and are associated with telomere defects[111]. Mutated genes causing secondary telomeropathies, when characterized, fall into DDR signaling pathways, DNA repair or nuclear lamina structure maintenance.

\subsection{DNA damage response:}

Ataxia-telangiectasia (AT) is an autosomal recessive neurodegenerative disorder with immunodeficiency, an increased risk of developing cancer in different tissues and ischemic heart diseases, caused by mutations in the ataxia-telangiectasia mutated (ATM) gene[112][113].

ATM, as well as its related kinase ATR (Ataxia-talangectasia mutated Rad3 related) and DNA PK, is a key transducer of DNA damage signaling, essential for repair. At telomeres, ATM and ATR kinases also coordinate telomerase recruitment in cancer cells to recover telomere length after replicative damage or telomere's dysfunction [114]. The essential function of protein kinases as a hub coordinating different damage signaling pathways (telomere attrition, oxidative stress, mitochondrial dysfunction) is emerging from mounting evidence in the literature (revised in [115]). Recently, the role of ATM in mediating telomere driven tissue inflammation has been directly assessed, revealing a telomere dysfunction-induced activation of the ATM-YAP1- pro-IL-18 pathway recruiting interferon (IFN)- $\gamma$-secreting T cells driving intestinal epithelium inflammation [116].

Mutations in proteins involved in telomere homeostasis have that have been recently linked to ATM activation suggesting that the number of secondary telomeropathies could be underestimated. Mutations in SMCHD1 cause facioscapulohumeral muscular dystrophy. SMCHD1 (structural maintenance of chromosomes flexible hinge domain containing protein 1 ) has been implicated in X-chromosome inactivation, imprinting and DNA damage repair. SMCHD1 deficient cells, display defects in ATM-dependent DNA checkpoint signaling at dysfunctional telomeres[117].

Nijmegen breakage syndrome (NBS) is a rare human disease displaying chromosome instability, radiosensitivity, cancer predisposition, immunodeficiency, and other defects. NBS is complexed with MRE11 and RAD50 in the NMR complex and is localized to telomere ends in association with TRF proteins. Blood cells from NBS patients have shortened telomere DNA ends [118]. Telomere length was reduced in homozygous and heterozygotes older than 30 years patients, in accordance with the respective cancer rates. [119]. 


\subsection{DNA repair}

Werner syndrome (WS), which is characterized by accelerated aging, is an autosomalrecessive genetic disorder caused by the mutation of Werner helicase (WRN). Hallmarks that define the aging process include genomic instability, telomere attrition, epigenetic alterations. Recent advances in WS research have elucidated WRN's role in DNA repair pathway choice regulation, telomere maintenance, resolution of complex DNA structures, epigenetic regulation, and stem cell maintenance [120]. WRN is a RECQ helicase involved in replication stress resolution by coordinating the enzymatic activity at single strand resection during homologous recombination and non-homologous end joining [121] [122][123][124].

Bloom syndrome, caused by biallelic mutations in BLM, is characterized by prenatal-onset growth deficiency, short stature, an erythematous photosensitive malar rash, and increased cancer predisposition. Diagnostically, a hallmark feature is the presence of increased sister chromatid exchanges (SCEs) on cytogenetic testing, and telomere attrition. Unresolved DNA recombination and/or replication intermediates persist into mitosis, leading to chromosome segregation defects and genome instability that most likely explain the growth restriction seen in these subjects and in Bloom syndrome[125]. Bloom syndrome is also associated with oxidative stress and mitochondrial dysfunction [126]. Bloom syndrome complex consists of the helicase BLM, the type IA topoisomerase TOP3A, and the OB-fold-containing RMI and RPA subunits. Each component contributes to function, interacts with each other, and the DNA that it manipulates/repairs. Biallelic mutations in TOP3A in ten individuals with prenatal-onset growth restriction and microcephaly. In addition, a homozygous truncating variant in RMI1, was found in two individuals with microcephalic dwarfism [127][128].

Fanconi anemia is a chromosomal instability syndrome characterized by increased frequency of chromosomal breakages, chromosomal radial figures, accelerated telomere shortening and telomere fusions. Patients with FA usually develop variable degrees of pancytopenia in childhood and often also have short stature and skin abnormalities. FA is caused by germline mutations in DNA repair genes of the FA/BRCA pathway[129].

\subsection{Nuclear envelope}

Progeroid laminopathies are induced by mutations in lamin A or in its interactors. Molecular mechanisms at the basis of laminopathies were studied mainly in the Hutchinson Gilford Progeria syndrome (HGPS), the best characterized progeroid laminopathy, and account for a role of lamin A in DNA metabolism and repair [130][131]. Telomere stabilization is dependent on the interaction with lamin A/C. TRF2 interacts with Lamin A but not with progerin, the truncated form of Lamin A causing progeroid syndromes[132]. Progerin expression reduces TRF2 stability and consequently leads to telomere attrition in atypical Werner syndrome[133]. The lamina-associated polypeptide 1 (LAP1) is also involved in lamins and chromatin positioning, and it was recently associated with telomeres function by TRF2 and RIF1 binding. However, the molecular mechanism through which LAP1 and TRF2 regulate telomere function remains to be elucidated[134]. 


\begin{tabular}{ccc}
\hline Disease & Molecular pathway & Mutated genes \\
\hline Ataxia talangectasia & DDR & ATM \\
\hline Facioscapulohumoral muscular dystrophy & DDR & NBS \\
\hline Nijmegen breakage syndrome & DDR & WRN \\
\hline Werner's syndrome & DNA repair & BLM/TOP3A/RMI \\
\hline Bloom's syndrome & DNA repair & FA/BRCA pathway \\
\hline Fanconi Anemia & DNA repair & LMNA/C \\
\hline Hutchinson Gilford Progeria syndrome & Nuclear envelope & FMR1 \\
\hline
\end{tabular}

\subsection{Undefined mechanisms}

The fragile $X$ premutation is defined by the expansion of the CGG trinucleotide repeat at the 5' UTR of the FMR1 gene. Men carrying a premutation are at increased risk for fragile $\mathrm{X}$-associated tremor/ataxia syndrome (FXTAS). It was recently demonstrated that premutation carriers had shorter telomeres compared with non-carriers, and that telomere shortening may be a consequence of the toxic effect of the premutation transcript with still undefined mechanisms[135].

\section{Conclusions}

Author Contributions: All authors have read and agreed to the published version of the manuscript.

Funding: This research was funded by AIRC \#17121 to E.S.

Conflicts of Interest: "The authors declare no conflict of interest."

\section{References}

[1] T. De Lange, "T-loops and the origin of telomeres," Nat. Rev. Mol. Cell Biol., vol. 5, no. 4, pp. 323-329, 2004, doi: 10.1038/nrm1359.

[2] R. J. Wellinger, "In the end, what's the problem?," Mol. Cell, vol. 53, no. 6, pp. 855-856, 2014, doi: 10.1016/j.molcel.2014.03.008.

[3] H. Xin et al., "TPP1 is a homologue of ciliate TEBP- $\beta$ and interacts with POT1 to recruit telomerase," Nature, vol. 445, no. 7127, pp. 559-562, 2007, doi: 10.1038/nature05469.

[4] L. Tomáška, A. J. Cesare, T. M. AlTurki, and J. D. Griffith, “Twenty years of t-loops: A case study for the importance of collaboration in molecular biology," DNA Repair (Amst)., vol. 94, no. June, p. 102901, 2020, doi: 10.1016/j.dnarep.2020.102901. 
[5] P. Ruis and S. J. Boulton, "The end protection problem - an unexpected twist in the tail," Genes Dev., vol. 35, no. 1-2, pp. 1-21, Jan. 2021, doi: 10.1101/gad.344044.120.

[6] E. M. Smith, D. F. Pendlebury, and J. Nandakumar, "Structural biology of telomeres and telomerase," Cellular and Molecular Life Sciences, vol. 77, no. 1. 2020, doi: 10.1007/s00018-019-03369-x.

[7] T. De Lange, "Shelterin: The protein complex that shapes and safeguards human telomeres," Genes Dev., vol. 19, no. 18, pp. 2100-2110, 2005, doi: 10.1101/gad.1346005.

[8] S. Hanaoka, A. Nagadoi, and Y. Nishimura, "Comparison between TRF2 and TRF1 of their telomeric DNAbound structures and DNA-binding activities," Protein Sci., vol. 14, no. 1, pp. 119-130, 2009, doi: 10.1110/ps.04983705.

[9] A. Sfeir and T. De Lange, "Removal of shelterin reveals the telomere end-protection problem," Science (80-. )., vol. 336, no. 6081, pp. 593-597, May 2012, doi: 10.1126/science.1218498.

[10] K. Cleal and D. M. Baird, "Catastrophic Endgames: Emerging Mechanisms of Telomere-Driven Genomic Instability," Trends in Genetics, vol. 36, no. 5. Elsevier Ltd, pp. 347-359, May 01, 2020, doi: 10.1016/j.tig.2020.02.001.

[11] J. W. Shay, "Role of telomeres and telomerase in aging and cancer," Cancer Discov., vol. 6, no. 6, pp. 584-593, 2016, doi: 10.1158/2159-8290.CD-16-0062.

[12] G. Aubert and P. M. Lansdorp, “Telomeres and aging," Physiological Reviews, vol. 88, no. 2. pp. 557-579, Apr. 2008, doi: 10.1152/physrev.00026.2007.

[13] D. Chakravarti, K. A. LaBella, and R. A. DePinho, “Telomeres: history, health, and hallmarks of aging.," Cell, Jan. 2021, doi: 10.1016/j.cell.2020.12.028.

[14] K. Demanelis et al., "Determinants of telomere length across human tissues," doi: 10.1126/science.aaz6876.

[15] A. Barral and J. Déjardin, "Telomeric Chromatin and TERRA," Journal of Molecular Biology, vol. 432, no. 15. Academic Press, pp. 4244-4256, Jul. 10, 2020, doi: 10.1016/j.jmb.2020.03.003.

[16] F. Rossiello et al., "DNA damage response inhibition at dysfunctional telomeres by modulation of telomeric DNA damage response RNAs," Nat. Commun., vol. 8, Feb. 2017, doi: 10.1038/ncomms13980.

[17] F. Michelini et al., "Damage-induced lncRNAs control the DNA damage response through interaction with DDRNAs at individual double-strand breaks," Nat. Cell Biol., vol. 19, no. 12, pp. 1400-1411, Nov. 2017, doi: 10.1038/ncb3643.

[18] J. Aguado et al., "Inhibition of DNA damage response at telomeres improves the detrimental phenotypes of Hutchinson-Gilford Progeria Syndrome," Nat. Commun., vol. 10, no. 1, Dec. 2019, doi: 10.1038/s41467-019-130183.

[19] E. Gilson and V. Géli, “How telomeres are replicated," Nature Reviews Molecular Cell Biology, vol. 8, no. 10. pp. 825-838, Oct. 2007, doi: 10.1038/nrm2259.

[20] E. Cusanelli and P. Chartrand, "Telomeric repeat-containing RNA TERRA: A noncoding RNA connecting telomere biology to genome integrity," Front. Genet., vol. 6, no. MAR, p. 143, 2015, doi: 10.3389/fgene.2015.00143.

[21] F. Rossiello, U. Herbig, M. P. Longhese, M. Fumagalli, and F. d'Adda di Fagagna, "Irreparable telomeric DNA damage and persistent DDR signalling as a shared causative mechanism of cellular senescence and ageing," Current Opinion in Genetics and Development, vol. 26. Elsevier Ltd, pp. 89-95, 2014, doi: 10.1016/j.gde.2014.06.009.

[22] A. L. Slusher, J. J. J. Kim, and A. T. Ludlow, "The role of alternative rna splicing in the regulation of htert, telomerase, and telomeres: Implications for cancer therapeutics," Cancers (Basel)., vol. 12, no. 6, pp. 1-16, 2020, doi: 10.3390/cancers12061514.

[23] K. Collins and J. R. Mitchell, "Telomerase in the human organism," Oncogene, vol. 21, no. 4 REV. ISS. 1, pp. $564-$ 579, 2002, doi: 10.1038/sj/onc/1205083.

[24] C. M. Roake and S. E. Artandi, "Regulation of human telomerase in homeostasis and disease," Nature Reviews Molecular Cell Biology, vol. 21, no. 7. Nature Research, pp. 384-397, Jul. 2020, doi: 10.1038/s41580-020-0234-z. 
[25] S. Victorelli and J. F. Passos, “Telomeres and Cell Senescence - Size Matters Not," EBioMedicine, vol. 21, pp. 1420, 2017, doi: 10.1016/j.ebiom.2017.03.027.

[26] M. I. Zvereva, D. M. Shcherbakova, and O. A. Dontsova, “Telomerase: Structure, functions, and activity regulation," Biochem., vol. 75, no. 13, pp. 1563-1583, 2010, doi: 10.1134/S0006297910130055.

[27] C. W. Greider and E. H. Blackburn, "Identification of a specific telomere terminal transferase activity in tetrahymena extracts," Cell, vol. 43, no. 2 PART 1, pp. 405-413, 1985, doi: 10.1016/0092-8674(85)90170-9.

[28] D. E. Gomez et al., "Telomere structure and telomerase in health and disease (Review)," Int. J. Oncol., vol. 41, no. 5, pp. 1561-1569, 2012, doi: 10.3892/ijo.2012.1611.

[29] J. L. Rinn, H. Y. Chang, and H. Y. Chang, "Long Noncoding RNAs: Molecular Modalities to Organismal Functions," Annu. Rev. Biochem., vol. 89, pp. 283-308, 2020, doi: 10.1146/annurev-biochem-062917-012708.

[30] H. D. M. Wyatt, S. C. West, and T. L. Beattie, "InTERTpreting telomerase structure and function," Nucleic Acids Res., vol. 38, no. 17, pp. 5609-5622, 2010, doi: 10.1093/nar/gkq370.

[31] A. Smogorzewska and T. De Lange, "Regulation of telomerase by telomeric proteins," Annual Review of Biochemistry, vol. 73. pp. 177-208, 2004, doi: 10.1146/annurev.biochem.73.071403.160049.

[32] J. D. Podlevsky and J. J. L. Chen, "It all comes together at the ends: Telomerase structure, function, and biogenesis," Mutat. Res. - Fundam. Mol. Mech. Mutagen., vol. 730, no. 1-2, pp. 3-11, 2012, doi: 10.1016/j.mrfmmm.2011.11.002.

[33] A. Angrisani, R. Vicidomini, M. Turano, and M. Furia, "Human dyskerin: Beyond telomeres," Biol. Chem., vol. 395, no. 6, pp. 593-610, 2014, doi: 10.1515/hsz-2013-0287.

[34] D. Hanahan and R. A. Weinberg, "The hallmarks of cancer," Cell, vol. 100, no. 1. Cell, pp. 57-70, Jan. 07, 2000, doi: 10.1016/S0092-8674(00)81683-9.

[35] F. P. Barthel et al., "Systematic analysis of telomere length and somatic alterations in 31 cancer types," Nat. Genet., vol. 49, no. 3, pp. 349-357, Mar. 2017, doi: 10.1038/ng.3781.

[36] A. Pestana, J. Vinagre, M. Sobrinho-Simões, and P. Soares, "TERT biology and function in cancer: Beyond immortalisation," Journal of Molecular Endocrinology, vol. 58, no. 2. BioScientifica Ltd., pp. R129-R146, Feb. 01, 2017, doi: 10.1530/JME-16-0195.

[37] S. G. Fernandes et al., "Role of telomeres and telomeric proteins in human malignancies and their therapeutic potential," Cancers, vol. 12, no. 7. MDPI AG, pp. 1-38, Jul. 01, 2020, doi: 10.3390/cancers12071901.

[38] N. Noureen et al., "Integrated analysis of telomerase enzymatic activity unravels an association with cancer stemness and proliferation.," Nat. Commun., vol. 12, no. 1, p. 139, Dec. 2021, doi: 10.1038/s41467-020-20474-9.

[39] D. Hockemeyer, A. J. Sfeir, J. W. Shay, W. E. Wright, and T. de Lange, “POT1 protects telomeres from a transient DNA damage response and determines how human chromosomes end," EMBO J., vol. 24, no. 14, pp. 2667-2678, Jul. 2005, doi: 10.1038/sj.emboj.7600733.

[40] J. B. Chaires et al., "Human POT1 unfolds G-quadruplexes by conformational selection," Nucleic Acids Res., vol. 48, no. 9, pp. 4976-4991, May 2020, doi: 10.1093/nar/gkaa202.

[41] Y. Wu, R. C. Poulos, and R. R. Reddel, "Role of pot1 in human cancer," Cancers (Basel)., vol. 12, no. 10, pp. 1-19, Oct. 2020, doi: 10.3390/cancers12102739.

[42] K. S. Butler, W. C. Hines, C. M. Heaphy, and J. K. Griffith, "Coordinate regulation between expression levels of telomere-binding proteins and telomere length in breast carcinomas," Cancer Med., vol. 1, no. 2, pp. 165-175, 2012, doi: 10.1002/cam4.14.

[43] M. Kirwan et al., "Dyskeratosis congenita and the DNA damage response," Br. J. Haematol., vol. 153, no. 5, pp. 634-643, 2011, doi: 10.1111/j.1365-2141.2011.08679.x.

[44] R. M. Marión et al., "Common Telomere Changes during In Vivo Reprogramming and Early Stages of Tumorigenesis," Stem Cell Reports, vol. 8, no. 2, pp. 460-475, Feb. 2017, doi: 10.1016/j.stemcr.2017.01.001. 
[45] H. Nan, A. A. Qureshi, J. Prescott, I. De Vivo, and J. Han, "Genetic variants in telomere-maintaining genes and skin cancer risk," Hum. Genet., vol. 129, no. 3, pp. 247-253, Mar. 2011, doi: 10.1007/s00439-010-0921-5.

[46] L. Bejarano et al., "Inhibition of TRF1 Telomere Protein Impairs Tumor Initiation and Progression in Glioblastoma Mouse Models and Patient-Derived Xenografts," Cancer Cell, vol. 32, no. 5, pp. 590-607.e4, Nov. 2017, doi: 10.1016/j.ccell.2017.10.006.

[47] P. Zizza et al., "TRF2 positively regulates SULF2 expression increasing VEGF - A release and activity in tumor microenvironment," Nucleic Acids Res., vol. 47, no. 7, 2019, doi: 10.1093/nar/gkz041.

[48] J. Cherfils-Vicini et al., "Cancer cells induce immune escape via glycocalyx changes controlled by the telomeric protein TRF 2 ," EMBO J., vol. 38, no. 11, 2019, doi: 10.15252/embj.2018100012.

[49] A. Biroccio et al., "TRF2 inhibits a cell-extrinsic pathway through which natural killer cells eliminate cancer cells," Nat. Cell Biol., vol. 15, no. 7, 2013, doi: 10.1038/ncb2774.

[50] M. El Maï et al., "The telomeric protein TRF2 regulates angiogenesis by binding and activating the PDGFR $\beta$ promoter," Cell Rep., vol. 9, no. 3, pp. 1047-1060, 2014, doi: 10.1016/j.celrep.2014.09.038.

[51] "Pan-cancer analyses reveal regulation and clinical outcome association of the shelterin complex in cancer PubMed." https://pubmed.ncbi.nlm.nih.gov/33497432/ (accessed Feb. 05, 2021).

[52] M. Adishesh et al., "Human endometrial carcinogenesis is associated with significant reduction in long noncoding rna, terra," Int. J. Mol. Sci., vol. 21, no. 22, pp. 1-13, Nov. 2020, doi: 10.3390/ijms21228686.

[53] B. Holohan, W. E. Wright, and J. W. Shay, "Telomeropathies: An emerging spectrum disorder," J. Cell Biol., vol. 205, no. 3, pp. 289-299, 2014, doi: 10.1083/jcb.201401012.

[54] R. G. Armando, D. L. Mengual Gomez, J. Maggio, M. C. Sanmartin, and D. E. Gomez, "Telomeropathies: Etiology, diagnosis, treatment and follow-up. Ethical and legal considerations," Clin. Genet., vol. 96, no. 1, pp. 3-16, 2019, doi: $10.1111 /$ cge.13526.

[55] B. P. Alter, P. S. Rosenberg, N. Giri, G. M. Baerlocher, P. M. Lansdorp, and S. A. Savage, "Telomere length is associated with disease severity and declines with age in dyskeratosis congenita.," Haematologica, vol. 97, no. 3, pp. 353-9, Mar. 2012, doi: 10.3324/haematol.2011.055269.

[56] A. Narla and B. L. Ebert, "Ribosomopathies: Human disorders of ribosome dysfunction," Blood, vol. 115, no. 16, pp. 3196-3205, 2010, doi: 10.1182/blood-2009-10-178129.

[57] D. Ruggero et al., "Dyskeratosis congenita and cancer in mice deficient in ribosomal RNA modification.," Science, vol. 299, no. 5604, pp. 259-62, Jan. 2003, doi: 10.1126/science.1079447.

[58] A. J. Walne and I. Dokal, “Dyskeratosis Congenita: A historical perspective," Mech. Ageing Dev., vol. 129, no. 12, pp. 48-59, 2008, doi: 10.1016/j.mad.2007.10.006.

[59] P. J. Mason and M. Bessler, "The genetics of dyskeratosis congenita," 2011, doi: 10.1016/j.cancergen.2011.11.002.

[60] I. Dokal, "Dyskeratosis congenita.," Hematology / the Education Program of the American Society of Hematology. American Society of Hematology. Education Program, vol. 2011. pp. 480-486, 2011, doi: 10.1182/asheducation2011.1.480.

[61] A. Aspesi and S. R. Ellis, "Rare ribosomopathies: insights into mechanisms of cancer," Nat. Rev. Cancer, vol. 19, no. 4, pp. 228-238, 2019, doi: 10.1038/s41568-019-0105-0.

[62] M. Armanios and E. H. Blackburn, “The telomere syndromes.," Nat. Rev. Genet., vol. 13, no. 10, pp. 693-704, 2012, doi: $10.1038 / \operatorname{nrg} 3246$.

[63] P. L. Opresko and J. W. Shay, “Telomere-associated aging disorders," Ageing Res. Rev., vol. 33, pp. 52-66, 2017, doi: 10.1016/j.arr.2016.05.009.

[64] S. A. Savage, "Dyskeratosis Congenita Summary Genetic counseling GeneReview Scope," pp. 1-30, 2020.

[65] B. P. Alter, N. Giri, S. A. Savage, and P. S. Rosenberg, "Cancer in dyskeratosis congenita," Blood, vol. 113, no. 26, pp. 6549-6557, 2009, doi: 10.1182/blood-2008-12-192880. 
[66] E. Balogh et al., "Pseudouridylation defect due to DKC1 and NOP10 mutations causes nephrotic syndrome with cataracts, hearing impairment, and enterocolitis," Proc. Natl. Acad. Sci. U. S. A., Jun. 2020, doi: 10.1073/pnas.2002328117.

[67] M. Penzo and L. Montanaro, “Turning uridines around: Role of rRNA pseudouridylation in ribosome biogenesis and ribosomal function," Biomolecules, vol. 8, no. 2. MDPI AG, Jun. 05, 2018, doi: 10.3390/biom8020038.

[68] N. Heiss, "X-linked dyskeratosis congenita is caused by mutations in a highly conserved gene with putative nucleolar functions," Nat. Genet., vol. 19, pp. 32-38, 1998.

[69] D. Nachmani et al., "Germline NPM1 mutations lead to altered rRNA 2'-O-methylation and cause dyskeratosis congenita," Nat. Genet., vol. 51, no. 10, pp. 1518-1529, 2019, doi: 10.1038/s41588-019-0502-z.

[70] T. Vulliamy et al., "Mutations in the telomerase component NHP2 cause the premature ageing syndrome dyskeratosis congenita.," Proc. Natl. Acad. Sci. U. S. A., vol. 105, no. 23, pp. 8073-8, Jun. 2008, doi: 10.1073/pnas.0800042105.

[71] J. S. Ibáñez-Cabellos, M. Seco-Cervera, C. Picher-Latorre, G. Pérez-Machado, J. L. García-Giménez, and F. V. Pallardó, "Acute depletion of telomerase components DKC1 and NOP10 induces oxidative stress and disrupts ribosomal biogenesis via NPM1 and activation of the P53 pathway," Biochim. Biophys. Acta - Mol. Cell Res., vol. 1867, no. 12, p. 118845, 2020, doi: 10.1016/j.bbamcr.2020.118845.

[72] S. Bergstrand et al., "Biallelic mutations in WRAP53 result in dysfunctional telomeres, Cajal bodies and DNA repair, thereby causing Hoyeraal-Hreidarsson syndrome," Cell Death Dis., vol. 11, no. 4, Apr. 2020, doi: 10.1038/s41419-020-2421-4.

[73] L. Y. Chen, S. Redon, and J. Lingner, "The human CST complex is a terminator of telomerase activity," Nature, vol. 488, no. 7412, pp. 540-544, 2012, doi: 10.1038/nature11269.

[74] M. P. Rubtsova et al., "Integrator is a key component of human telomerase RNA biogenesis," Sci. Rep., vol. 9, no. 1, pp. 1-10, 2019, doi: 10.1038/s41598-018-38297-6.

[75] M. Benyelles et al., "Impaired telomere integrity and rRNA biogenesis in PARN-deficient patients and knockout models," EMBO Mol. Med., vol. 11, no. 7, Jul. 2019, doi: 10.15252/emmm.201810201.

[76] H. Kocak et al., "Hoyeraal-Hreidarsson syndrome caused by a germline mutation in the TEL patch of the telomere protein TPP1," Genes Dev., vol. 28, no. 19, pp. 2090-2102, Oct. 2014, doi: 10.1101/gad.248567.114.

[77] A. Witkowska et al., "Downregulation of telomerase maintenance-related acd expression in patients undergoing immunosuppresive therapy following kidney transplantation," Exp. Ther. Med., vol. 10, no. 6, pp. 2224-2230, 2015, doi: 10.3892/etm.2015.2785.

[78] M. S. O'Connor, A. Safari, H. Xin, D. Liu, and Z. Songyang, "A critical role for TPP1 and TIN2 interaction in high-order telomeric complex assembly," Proc. Natl. Acad. Sci. U. S. A., vol. 103, no. 32, pp. 11874-11879, 2006, doi: 10.1073/pnas.0605303103.

[79] S. A. Savage, N. Giri, G. M. Baerlocher, N. Orr, P. M. Lansdorp, and B. P. Alter, "TINF2, a Component of the Shelterin Telomere Protection Complex, Is Mutated in Dyskeratosis Congenita," Am. J. Hum. Genet., vol. 82, no. 2, pp. 501-509, 2008, doi: 10.1016/j.ajhg.2007.10.004.

[80] M. Okuwaki, S. Saito, H. Hirawake-Mogi, and K. Nagata, “The interaction between nucleophosmin/NPM1 and the large ribosomal subunit precursors contribute to maintaining the nucleolar structure," Biochim. Biophys. Acta - Mol. Cell Res., vol. 1868, no. 1, p. 118879, 2021, doi: 10.1016/j.bbamcr.2020.118879.

[81] W. E. Wright, M. A. Piatyszek, W. E. Rainey, W. Byrd, and J. W. Shgy, “Telomerase Activity in Human Germline and Embryonic Tissues and Cells," vol. 179, 1996.

[82] E. Aix, Ó. Gutiérrez-Gutiérrez, C. Sánchez-Ferrer, T. Aguado, and I. Flores, "Postnatal telomere dysfunction induces cardiomyocyte cell-cycle arrest through p21 activation," J. Cell Biol., vol. 213, no. 5, pp. 571-583, 2016, doi: 10.1083/jcb.201510091. 
[83] C. Sucularli, P. Thomas, H. Kocak, J. S. White, B. C. O'Connor, and C. E. Keegan, “High-throughput gene expression analysis identifies p53-dependent and -independent pathways contributing to the adrenocortical dysplasia (acd) phenotype," Gene, vol. 679, pp. 219-231, 2018, doi: 10.1016/j.gene.2018.09.002.

[84] S. S. Jose, F. Tidu, P. Burilova, T. Kepak, K. Bendickova, and J. Fric, “The telomerase complex directly controls hematopoietic stem cell differentiation and senescence in an induced pluripotent stem cell model of telomeropathy," Front. Genet., vol. 9, no. AUG, Aug. 2018, doi: 10.3389/fgene.2018.00345.

[85] M. M. Gramatges and A. A. Bertuch, "Short Telomeres: From Dyskeratosis Congenita to Sporadic Aplastic Anemia and Malignancy," Transl Res, vol. 162, no. 6, 2013, doi: 10.1016/j.trsl.2013.05.003.

[86] J. Pelletier, G. Thomas, and S. Volarevic, "Players and Therapeutic Avenues," Nat. Publ. Gr., vol. 18, no. 1, pp. 51-63, 2017, [Online]. Available: http://dx.doi.org/10.1038/nrc.2017.104.

[87] D. O. Warmerdam and R. M. F. Wolthuis, "Keeping ribosomal DNA intact: a repeating challenge," Chromosom. Res., vol. 27, no. 1-2, pp. 57-72, 2019, doi: 10.1007/s10577-018-9594-z.

[88] S. Ojha, S. Malla, and S. M. Lyons, "SnoRNPs: Functions in ribosome biogenesis," Biomolecules, vol. 10 , no. 5. MDPI AG, May 01, 2020, doi: 10.3390/biom10050783.

[89] M. Tsekrekou, K. Stratigi, and G. Chatzinikolaou, "The nucleolus: In genome maintenance and repair," Int. J. Mol. Sci., vol. 18, no. 7, 2017, doi: 10.3390/ijms18071411.

[90] J. R. Mitchell, E. Wood, and K. Collins, "A telomerase component is defective in the human disease dyskeratosis congenita," Nature, vol. 402, no. 6761, pp. 551-555, 1999, doi: 10.1038/990141.

[91] S. G. Jayakrishnan Nandakumar, “Molecular mechanisms of telomere biology disorders,” J. Biol. Chem., 2020, doi: 10.1074/jbc.REV120.014017.

[92] F. L. Zhong, L. F. Z. Batista, A. Freund, M. F. Pech, A. S. Venteicher, and S. E. Artandi, “TPP1 OB-fold domain controls telomere maintenance by recruiting telomerase to chromosome ends," Cell, vol. 150, no. 3, pp. 481-494, 2012, doi: 10.1016/j.cell.2012.07.012.

[93] E. Abreu et al., “TIN2-Tethered TPP1 Recruits Human Telomerase to Telomeres In Vivo," Mol. Cell. Biol., vol. 30, no. 12, pp. 2971-2982, 2010, doi: 10.1128/mcb.00240-10.

[94] P. Veverka, T. Janovič, and C. Hofr, “Quantitative biology of human shelterin and telomerase: Searching for the weakest point," Int. J. Mol. Sci., vol. 20, no. 13, 2019, doi: 10.3390/ijms20133186.

[95] S. Burra et al., "Human AP-endonuclease (Ape1) activity on telomeric G4 structures is modulated by acetylatable lysine residues in the N-terminal sequence," pp. 129-143, 2019, doi: 10.1016/j.dnarep.2018.11.010.Human.

[96] A. K. Mukherjee et al., "Telomere length-dependent transcription and epigenetic modifications in promoters remote from telomere ends," PLoS Genet., vol. 14, no. 11, pp. 1-24, 2018, doi: 10.1371/journal.pgen.1007782.

[97] F. M. Boisvert, S. Van Koningsbruggen, J. Navascués, and A. I. Lamond, "The multifunctional nucleolus," Nat. Rev. Mol. Cell Biol., vol. 8, no. 7, pp. 574-585, 2007, doi: 10.1038/nrm2184.

[98] S. Zhang, P. Hemmerich, and F. Grosse, "Nucleolar localization of the human telomeric repeat binding factor 2 (TRF2)," J. Cell Sci., vol. 117, no. 17, pp. 3935-3945, 2004, doi: 10.1242/jcs.01249.

[99] E. T. Stoopler and R. M. Shanti, “Dyskeratosis Congenita,” Mayo Clin. Proc., vol. 94, no. 9, pp. 1668-1669, 2019, doi: 10.1016/j.mayocp.2019.04.032.

[100] T. P. B. Handley, J. A. McCaul, and G. R. Ogden, “The diagnosis and treatment of dyskeratosis congenita: a review," Oral Oncol., vol. 42, no. 4, pp. 331-336, 2006, doi: 10.1016/j.oraloncology.2005.06.007.

[101] A. L. Kennedy and A. Shimamura, “Genetic predisposition to MDS: Clinical features and clonal evolution,” Blood, vol. 133, no. 10, pp. 1071-1085, 2019, doi: 10.1182/blood-2018-10-844662.

[102] J. Feldstein and S. Fernandez Garcia, “The diagnosis and treatment of dyskeratosis congenita: a review," J. Blood Med., vol. Volume 201, no. 5, p. 157, Aug. 2014, doi: 10.2147/JBM.S47437.

[103] S. Agarwal, "Evaluation and Management of Hematopoietic Failure in Dyskeratosis Congenita," Hematol. Oncol. 
Clin. North Am., vol. 32, no. 4, pp. 669-685, 2018, doi: 10.1016/j.hoc.2018.04.003.

[104] M. F. Gomes, P. P. De Abreu, É. C. D. F. Banzi, and T. D. O. Nogueira, “Interdisciplinary approach to treat dyskeratosis congenita associated with severe aplastic anemia: A case report," Spec. Care Dent., vol. 26, no. 2, pp. 81-84, 2006, doi: 10.1111/j.1754-4505.2006.tb01515.x.

[105] M. R. Niewisch and S. A. Savage, "An update on the biology and management of dyskeratosis congenita and related telomere biology disorders," Expert Review of Hematology, vol. 12, no. 12. Taylor and Francis Ltd, pp. 10371052, Dec. 02, 2019, doi: 10.1080/17474086.2019.1662720.

[106] S. Agarwal et al., "Telomere elongation in induced pluripotent stem cells from dyskeratosis congenita patients," vol. 464, no. 7286, pp. 292-296, 2010, doi: 10.1038/nature08792.Telomere.

[107] A. A. Mangaonkar and M. M. Patnaik, "Short Telomere Syndromes in Clinical Practice: Bridging Bench and Bedside," Mayo Clin. Proc., vol. 93, no. 7, pp. 904-916, 2018, doi: 10.1016/j.mayocp.2018.03.020.

[108] A. Chalkiadaki and L. Guarente, "The multifaceted functions of sirtuins in cancer," Nat. Rev. Cancer, vol. 15, no. 10, pp. 608-624, 2015, doi: 10.1038/nrc3985.

[109] M. Serravallo, J. Jagdeo, S. A. Glick, D. M. Siegel, and N. I. Brody, "Sirtuins in dermatology: Applications for future research and therapeutics," Arch. Dermatol. Res., vol. 305, no. 4, pp. 269-282, 2013, doi: 10.1007/s00403-013$1320-2$.

[110] A. Rizzo et al., "SIRT6 interacts with TRF2 and promotes its degradation in response to DNA damage," vol. 45, no. 4, pp. 1820-1834, 2017, doi: 10.1093/nar/gkw1202.

[111] P. L. Opresko and J. W. Shay, "Telomere-associated aging disorders," Ageing Research Reviews, vol. 33. Elsevier Ireland Ltd, pp. 52-66, Jan. 01, 2017, doi: 10.1016/j.arr.2016.05.009.

[112] N. J. H. van Os et al., "Health risks for ataxia-telangiectasia mutated heterozygotes: a systematic review, metaanalysis and evidence-based guideline," Clinical Genetics, vol. 90, no. 2. Blackwell Publishing Ltd, pp. 105-117, Aug. 01, 2016, doi: 10.1111/cge.12710.

[113] A. L. Renault et al., "Telomere length, ATM mutation status and cancer risk in Ataxia-Telangiectasia families," Carcinogenesis, vol. 38, no. 10, pp. 994-1003, Oct. 2017, doi: 10.1093/carcin/bgx074.

[114] A. S. Tong et al., "ATM and ATR Signaling Regulate the Recruitment of Human Telomerase to Telomeres," Cell Rep., vol. 13, no. 8, pp. 1633-1646, Nov. 2015, doi: 10.1016/j.celrep.2015.10.041.

[115] A. B. Engin and A. Engin, "Aging and Protein Kinases," Springer, Cham, 2021, pp. 35-69.

[116] D. Chakravarti et al., "Telomere dysfunction activates YAP1 to drive tissue inflammation," Nat. Commun., vol. 11, no. 1, Dec. 2020, doi: 10.1038/s41467-020-18420-w.

[117] A. Vančevska, W. Ahmed, V. Pfeiffer, M. Feretzaki, S. J. Boulton, and J. Lingner, “ SMCHD 1 promotes ATM dependent DNA damage signaling and repair of uncapped telomeres ," EMBO J., vol. 39, no. 7, Apr. 2020, doi: 10.15252/embj.2019102668.

[118] V. Ranganathan et al., "Rescue of a telomere length defect of Nijmegen breakage syndrome cells requires NBS and telomerase catalytic subunit," Curr. Biol., vol. 11, no. 12, pp. 962-966, Jun. 2001, doi: 10.1016/S09609822(01)00267-6.

[119] R. Habib et al., "Telomere attrition and dysfunction: A potential trigger of the progeroid phenotype in nijmegen breakage syndrome," Aging (Albany. NY)., vol. 12, no. 12, pp. 12342-12375, Jun. 2020, doi: 10.18632/aging.103453.

[120] J. Oshima, J. M. Sidorova, and R. J. Monnat, "Werner syndrome: Clinical features, pathogenesis and potential therapeutic interventions," Ageing Research Reviews, vol. 33, no. 5. Elsevier Ireland Ltd, pp. 105-114, Jan. 01, 2017, doi: 10.1016/j.arr.2016.03.002.

[121] P. Pichierri, F. Ammazzalorso, M. Bignami, and A. Franchitto, "The Werner Syndrome protein: Linking the replication checkpoint response to genome stability," Aging (Albany. NY)., vol. 3, no. 3, pp. 311-318, 2011, doi: 10.18632/aging.100293. 
[122] V. Palermo et al., "CDK1 phosphorylates WRN at collapsed replication forks," Nat. Commun., vol. 7, Sep. 2016, doi: $10.1038 /$ ncomms12880.

[123] C. Mukherjee et al., "RIF1 promotes replication fork protection and efficient restart to maintain genome stability," Nat. Commun., vol. 10, no. 1, Dec. 2019, doi: 10.1038/s41467-019-11246-1.

[124] J. Garzón, S. Ursich, M. Lopes, S. ichiro Hiraga, and A. D. Donaldson, “Human RIF1-Protein Phosphatase 1 Prevents Degradation and Breakage of Nascent DNA on Replication Stalling," Cell Rep., vol. 27, no. 9, pp. 25582566.e4, May 2019, doi: 10.1016/j.celrep.2019.05.002.

[125] A. M. K. Shorrocks et al., "The Bloom syndrome complex senses RPA-coated single-stranded DNA to restart stalled replication forks," Nat. Commun., vol. 12, no. 1, Dec. 2021, doi: 10.1038/s41467-020-20818-5.

[126] V. Subramanian, B. Rodemoyer, V. Shastri, L. J. Rasmussen, C. Desler, and K. H. Schmidt, “Bloom syndrome DNA helicase deficiency is associated with oxidative stress and mitochondrial network changes.," Sci. Rep., vol. 11, no. 1, p. 2157, Jan. 2021, doi: 10.1038/s41598-021-81075-0.

[127] C. A. Martin et al., "Mutations in TOP3A Cause a Bloom Syndrome-like Disorder," Am. J. Hum. Genet., vol. 103, no. 2, pp. 221-231, Aug. 2018, doi: 10.1016/j.ajhg.2018.07.001.

[128] R. Bythell-Douglas and A. J. Deans, "A Structural Guide to the Bloom Syndrome Complex," Structure, vol. 29, no. 2. Cell Press, pp. 99-113, Feb. 04, 2021, doi: 10.1016/j.str.2020.11.020.

[129] J. Filipović et al., "First molecular-cytogenetic characterization of Fanconi anemia fragile sites in primary lymphocytes of FA-D2 patients in different stages of the disease," Mol. Cytogenet., vol. 9, no. 1, Sep. 2016, doi: 10.1186/s13039-016-0280-6.

[130] R. Burla, M. La Torre, C. Merigliano, F. Vernì, and I. Saggio, “Genomic instability and DNA replication defects in progeroid syndromes," Nucleus, vol. 9, no. 1, pp. 368-379, Jan. 2018, doi: 10.1080/19491034.2018.1476793.

[131] F. Martins, J. Sousa, C. D. Pereira, O. A. B. da Cruz e Silva, and S. Rebelo, “Nuclear envelope dysfunction and its contribution to the aging process," Aging Cell, vol. 19, no. 5. Blackwell Publishing Ltd, p. e13143, May 01, 2020, doi: 10.1111/acel.13143.

[132] A. M. Wood et al., "TRF2 and lamin A/C interact to facilitate the functional organization of chromosome ends," Nat. Commun., vol. 5, 2014, doi: 10.1038/ncomms6467.

[133] B. Saha et al., "DNA damage accumulation and TRF2 degradation in atypical Werner syndrome fibroblasts with LMNA mutations.," Front. Genet., vol. 4, no. JUL, p. 129, 2013, doi: 10.3389/fgene.2013.00129.

[134] J. B. Serrano, O. A. B. da Cruz E Silva, and S. Rebelo, "Lamina Associated Polypeptide 1 (LAP1) Interactome and Its Functional Features.," Membranes (Basel)., vol. 6, no. 1, Jan. 2016, doi: 10.3390/membranes6010008.

[135] I. Albizua, P. Chopra, E. G. Allen, W. He, A. S. Amin, and S. L. Sherman, "Study of telomere length in men who carry a fragile X premutation or full mutation allele," Hum. Genet., vol. 139, no. 12, pp. 1531-1539, Dec. 2020, doi: 10.1007/s00439-020-02194-8. 